\title{
STC-1 ameliorates renal injury in diabetic nephropathy by inhibiting the expression of BNIP3 through the AMPK/SIRT3 pathway
}

\author{
Zhiwen $\mathrm{Liu}^{1} \cdot$ Hong $\mathrm{Liu}^{1} \cdot \mathrm{Li} \mathrm{Xiao}^{1} \cdot$ Guoyong $\mathrm{Liu}^{2} \cdot \operatorname{Lin~Sun}^{1} \cdot \operatorname{Liyu} \mathrm{He} \mathbb{C D}^{1}$
}

Received: 22 June 2018 / Revised: 10 October 2018 / Accepted: 22 October 2018 / Published online: 25 January 2019

(c) United States \& Canadian Academy of Pathology 2019

\begin{abstract}
Diabetic kidney disease (DKD) is the leading cause of morbidity and mortality in individuals with diabetes, and it is the leading cause of end-stage renal disease (ESRD) worldwide. Stanniocalcin-1 (STC-1) is present in various tissues, and it has antioxidant and anti-apoptotic activities, which play a role in kidney protection, including diabetic nephropathy (DN). However, the mechanism that underlies these effects remains unknown. This study suggests that STC-1 ameliorates oxidative stress and cell apoptosis in the kidneys of $\mathrm{db} / \mathrm{db}$ mice and high glucose (HG)-treated BUMPT cells by inhibiting Bnip3 expression through AMPK/Sirt3 pathway activation. In the clinic, DKD patients with high levels of STC-1 have a better prognosis than patients with low STC-1 levels. Thus, we concluded that STC-1 ameliorates renal injury in DN by inhibiting the expression of Bnip3 through the AMPK/SIRT3 pathway and that serum STC-1 is independently associated with DKD progression in patients with type 2 diabetes. As high STC-1 levels indicate a better prognosis, synthetic STC-1 may become a potential drug for the treatment of DKD patients.
\end{abstract}

\section{Introduction}

Diabetic kidney disease (DKD) is the leading cause of morbidity and mortality in individuals with diabetes, and it is the leading cause of end-stage renal disease (ESRD) worldwide [1]. DKD has traditionally been diagnosed on the basis of persistent proteinuria and impaired renal function [2]. The pathogenesis of DKD remains unclear; however, a substantial number of causes are considered to be related to the development of DKD, including oxidative stress and apoptosis $[3,4]$.

Stanniocalcin-1 (STC-1) is present in various tissues and is thought to be an autocrine or paracrine factor [5]. STC-1 is expressed in many cells and can be released into the extracellular milieu; moreover, it exerts its

Liyu He

heliyu1124@csu.edu.cn

1 Department of Nephrology, The Second Xiangya Hospital, Central South University, Key Lab of Kidney Disease and Blood Purification in Hunan, 139 Renmin Road, Changsha, Hunan 410011, People's Republic of China

2 Department of Nephrology, The First Affiliated Hospital of Changde Vocational Technical College, Changde 415000, People's Republic of China physiological role by binding to proteins on the cell surface or the mitochondrial membrane [6]. STC-1 was originally thought to be the glycoprotein hormone that regulates the dynamic balance of calcium and phosphate in fish [7]. However, in recent studies, it has been reported that STC-1 has antioxidant, anti-inflammatory, and antiapoptotic activities, which play a role in kidney protection, including diabetic nephropathy (DN) [8]. STC-1 inhibits renal ischemia/reperfusion injury via an AMPactivated protein kinase (AMPK)-dependent pathway; moreover, they reported that AMPK regulates the expression of UCP2 and sirtuin 3 (Sirt3) induced by STC1 , resulting in kidney protection, which suggests that the STC-1/AMPK/Sirt3 signaling pathway plays a critical role in ischemia/reperfusion-induced kidney injury [9]. Sirt3 is the main deacetylase in the mitochondrial matrix that promotes aerobic metabolism, and it is the instrumental regulator of ROS [10]. ROS production contributes to DN development, and a lack of Sirt3 aggravates tissue damage caused by ROS [11]. Thus, we hypothesized that STC-1 delays the progression of DN through the inhibition of ROS production via an AMPK/Sitr3related signaling pathway.

Bcl-2-like 19-kDa-interacting protein 3 (Bnip3), a member of the Bcl2 family, is a critical regulator of mitochondrial function and cell apoptosis [12, 13]. The 
overexpression of Bnip3 leads to the opening of the mitochondrial permeability transformation pore (PTP), thereby eliminating the proton electrochemical gradient, which activates a series of events that ultimately lead to chromatin concentration and DNA fragmentation [14]. Regula KM et al. demonstrated that Bnip3 provokes mitochondrial defects and cell death in hypoxia [15]. In addition, the inhibition of Bnip3 protects mitochondrial function and reduces cell death $[16,17]$. ROS and cell death are highly related to the progression of DN. Thus, the inhibition of oxidative stress and apoptosis can delay the development of DN. Interestingly, numerous studies have reported that both ROS production and cell death are related to the expression of Bnip3, indicating that Bnip3 may be a potential therapeutic target for DN $[18,19]$. Qiong Du et al. found that Sirt3 attenuates doxorubicin-induced cardiac hypertrophy and mitochondrial dysfunction via the suppression of Bnip3. These findings suggest a new molecular mechanism in which Sirt3 restores the mitochondrial respiratory chain defects by inhibiting the expression of Bnip3, indicating a potential relationship between Sirt3 and Bnip3 [20].

Given the previously described findings, it is plausible that the AMPK/Sirt3/Bnip3 signaling pathway plays a critical role in DN development. In this study, we observed an altered expression of STC-1 in the serum of patients with different degrees of DN and their prognosis. Moreover, we assessed the relationship between the serum STC-1 level and prognosis in DN patients. Finally, we analyzed the expression of Sirt3, Bnip3, and AMPK both in vivo and in vitro. Together, our findings suggest that STC-1 ameliorates renal injury in DN by inhibiting the expression of Bnip3 through the AMPK/SIRT3 pathway.

\section{Materials and methods}

\section{Basic medical research}

\section{Animal experimental design}

Twelve-week-old male C57BL/6J $\mathrm{db} / \mathrm{db}$ and C57BL/6J $\mathrm{db} / \mathrm{m}$ mice were used for the animal experiments. These mice were purchased from the JunKe Experimental Animal Company (Nanjing, China). Mice were organized into the following three groups for the animal experiments: the $\mathrm{db} / \mathrm{m}$ group (control, $n=6$ ); the $\mathrm{db} / \mathrm{db}$ group $(n=8)$; and the $\mathrm{db} / \mathrm{db}$ group that received an intraperitoneal injection (i.p.) of rSTC-1 (BioVendor Research and Diagnostic Products, Asheville, NC or Czech Republic, $n=8$ ). The mice were euthanized at 24 weeks of age. The Animal Care and Use Committee of Second Xiangya Hospital of Central South University approved all animal procedures.

\section{Morphological studies}

Kidney tissues were fixed with $4 \%$ buffered paraformaldehyde and were embedded in paraffin; 4- $\mu$ m-thick sections were subsequently prepared. The sections were then subjected to periodic acid-Schiff (PAS) and Masson's trichrome staining. A semiquantitative scoring system was used to evaluate the tubulointerstitial injury severity.

\section{Immunohistochemical and immunofluorescence staining}

Immunofluorescence and immunohistochemical stains were performed on formalin-fixed, paraffin-embedded 4- $\mu \mathrm{m}$ sections. The sections were rehydrated, and antigens were retrieved using heated citrate. For immunohistochemistry, staining was visualized using horseradish peroxidasecoupled secondary antibodies (Vectastain Elite; Vector Laboratories, Peterborough, UK). For immunofluorescence, the slides were exposed to FITC- (1:200) or rhodaminelabeled (1:500) secondary antibodies (Jackson ImmunoResearch, West Grove, PA, USA). The sections were mounted in Vectashield medium that contained DAPI (Invitrogen, Carlsbad, CA, USA). Images were acquired using a fluorescence microscope (Nikon, Tokyo, Japan). Related isotype immunoglobulins (Jackson ImmunoResearch) were used as negative controls for all stainings. All immunohistochemical and immunofluorescence analyses were repeated at least three times, and representative images are presented.

\section{Measurement of ROS generation and apoptosis}

Dihydroethidium (DHE) was used to assess the production of intracellular superoxide anion $\left(\mathrm{O}_{2-}\right)$. TUNEL staining was used to evaluate apoptosis following the manufacturer's instructions (Roche, Basel, Switzerland).

\section{Immunoassays}

Superoxide dismutase (SOD), malondialdehyde (MDA), and STC-1 were measured by an ELISA kit (R\&D Systems) according to the manufacturer's instructions.

\section{Apoptosis assessment by Annexin V-FITC and propidium iodide staining}

Cell apoptosis was measured by Annexin V FACS according to the manufacturer's protocol (Calbiochem). Briefly, cells were harvested after the incubation period and were washed in cold PBS; the cells were subsequently incubated in $300 \mu \mathrm{l}$ of binding buffer that contained $3 \mu \mathrm{l}$ of Annexin V-FITC and $3 \mu \mathrm{l}$ of propidium iodide in the dark for $15 \mathrm{~min}$ at room temperature. The stained samples (which contained 200,000 cells/sample) were subsequently analyzed on a FACS Calibur 
Table 1 Specific grouping

\begin{tabular}{lllllll}
\hline & \multicolumn{2}{l}{ ACR $<30 \mathrm{mg} / \mathrm{g}$} & \multicolumn{2}{l}{$30 \mathrm{mg} / \mathrm{g} \leq \mathrm{ACR}<$} & \multicolumn{2}{l}{ ACR $\geq 300 \mathrm{mg} / \mathrm{g}$} \\
& Low & High & Low & High & Low & High \\
Number & 99 & 117 & 112 & 91 & 140 & 132 \\
Sex (male/female) & $49 / 50$ & $56 / 61$ & $60 / 52$ & $48 / 43$ & $75 / 65$ & $62 / 70$ \\
Age & $51.0 \pm 7.4$ & $50 . \pm 9.1$ & $53.6 \pm 6.8$ & $54.9 \pm 7.1$ & $55.1 \pm 6.0$ & $57.8 \pm 7.3$ \\
\hline
\end{tabular}

flow cytometer within $1 \mathrm{~h}$ following the manufacturer's protocol (BD Biosciences) [21].

\section{Assessment of physiological features and renal function}

Body weights were measured, and blood, serum, and urine sample collection was performed every 2 weeks. The blood glucose levels were detected using a blood glucose monitor (Boehringer Mannheim, Mannheim, Germany). The urine albumin concentrations were measured using a mouse urine albumin ELISA kit (Bethyl Laboratories, USA), and the serum creatinine levels were assessed with a QuantiChrom Creatinine Assay Kit (BioAssay Systems, USA) according to the manufacturer's protocol. Urinary ACR was calculated as the urine albumin/creatinine ratio. The urine $\beta-\mathrm{NAG}$ was measured using an automated colorimetric method (Pacific Biomarkers, Inc., USA).

\section{Cell culture studies and reagents}

The Boston University mouse proximal tubular cell line (BUMPT) was used in this study (obtained from the Dong Lab). BUMPT cells were plated in 35- $\mathrm{mm}$ dishes at a density of $0.3 \times 10^{6}$ cells/dish. The cells were starved overnight in serum-free DMEM medium supplemented with $10 \%$ FBS, insulin $(10 \mu \mathrm{g} / \mathrm{ml})$, transferrin $(5 \mu \mathrm{g} / \mathrm{ml})$, epidermal growth factor $(10 \mathrm{ng} / \mathrm{ml})$, and dexamethasone $(4 \mu \mathrm{g} / \mathrm{ml})$. The cells were treated with $35 \mathrm{mM}$ glucose for the indicated time. The experimental group was pretreated with $\mathrm{rSTC}-1(1.0 \mu \mathrm{g} / \mathrm{ml})$ [22]. Control cells were maintained in normal medium. After treatment, the cells were further cultured for $24 \mathrm{~h}$; the cells were monitored morphologically or collected for biochemical analyses [23]. Cell-permeable activator of AMP-activated protein kinase (AICAR, $500 \mu \mathrm{M})$, a selective and reversible AMP-kinase inhibitor (dorsomorphin, $10 \mu \mathrm{M}$ ), and a selective SIRT3 inhibitor (3-TYP, $50 \mu \mathrm{M})$ were purchased from Abcam (UK). The doses of AICAR, dorsomorphin, and YC-1 were selected on the basis of existing data [24-27].

\section{Cell immunofluorescence}

Cells were grown on coverslips, washed three times with PBS, fixed in $4 \%$ paraformaldehyde for $20 \mathrm{~min}$, permeabilized with $0.1 \%$ Triton $\mathrm{X}-100$, and then incubated in blocking buffer. The cells were subsequently incubated in primary antibody solution overnight. The cells were then incubated with FITC- or rhodamine-conjugated secondary antibodies and examined with a Zeiss fluorescence microscope equipped with U Vepi-illumination [28].

\section{Extraction of total RNA and quantitative real-time PCR}

Total RNA was isolated from the kidneys of individual mice using TRIzol (TaKaRa, Dalian, China). cDNA was synthesized using the M-MLV Reverse Transcriptase cDNA Synthesis Kit (TaKaRa) according to the manufacturer's instructions. Real-time PCR was performed with an ABI Prism 7300 Sequence Detection system (Applied Biosystems) using SYBR ${ }^{\circledast}$ Premix Ex Taq TM II (TaKaRa). The primer sequences are shown in Table 1. The gene expression in each sample was analyzed in duplicate and normalized against the internal control gene (GAPDH). Relative quantification of the target gene expression in patients compared with normal samples was performed via the $\Delta \Delta \mathrm{Ct}$ method [29].

\section{Western blotting}

Lysates of kidney tissue or BUMPT cells were prepared. All antibodies used for western blotting were purchased from Cell Signaling Technology Inc. (Beverly, MA, USA), and the diluted concentration was based on the antibody instructions. The membranes were incubated with primary antibodies overnight at $4{ }^{\circ} \mathrm{C}$ and washed with TBST three times. Secondary horseradish peroxidase-conjugated antibodies $(1: 10,000)$ were incubated with the membranes for $1 \mathrm{~h}$ at room temperature. Antibody-positive bands were visualized using a VersaDoc imaging system (Bio-Rad, USA). Data were analyzed by Quantity One (Bio-Rad, USA). Peroxidase and bands were visualized using the Super Western Sensitivity Enhanced Chemiluminescence Detection System (ECL kit, Pierce Biotechnology, Pierce, IL, USA). Image J was used to quantify the density of the bands.

\section{Prospective cohort study}

\section{Inclusion criteria and exclusion criteria}

Inclusion criteria: (1) patients diagnosed with type 2 diabetes according to the 2005 Diabetes Prevention Guidelines in China; (2) age of 45-60 years; (3) willing to sign an 
informed consent; and (4) patient occupation fixed and able to provide detailed information.

Exclusion criteria: (1) patients diagnosed with other diseases that cause increased urinary albumin; (2) patients diagnosed with severe brain, heart, or liver diseases; or (3) patients diagnosed with severe mental disorders.

According to the previously described criteria, we recruited 691 patients from the Second Xiangya Hospital of Central South University during 2012-2013. These patients were divided into three groups based on the ACR value as follows: normal to mildly elevated (ACR $<30 \mathrm{mg} / \mathrm{g}$, "normoalbuminuria"), moderately elevated (ACR30-299 mg/g, "microalbuminuria"), or severely elevated (ACR $>300 \mathrm{mg} / \mathrm{g}$, "macroalbuminuria") [30]. In each group, the serum STC-1 levels were measured using ELISA kits. Each group was divided into two subgroups according to the STC-1 level. The mean STC-1 level was calculated in each group. STC-1 levels less than the mean were defined as the low group, and STC-1 levels greater than the mean were assigned to the high group. The specific grouping is shown in Table 1.

Within each group, there were no significant differences between the low and high groups with respect to age and sex. Moreover, each patient had strictly controlled blood glucose during the follow-up.

\section{Follow-up methods}

Through various methods (mail, mobile phone, telephone, email, and fax), the patients were contacted to make regular appointments at the hospital for follow-up. The center assigned trained personnel to determine the patient's condition, treatment, and examination. The follow-up observation time points were 12,24 , and 36 months after enrollment, and the follow-up was completed on January 1, 2016.

\section{Outcomes}

If the following conditions occurred during the follow-up, the endpoint event was calculated using the following equation:

1. ACR progression (normal- to micro- or macroalbuminuria; micro- to macroalbuminuria)

2. Receiving dialysis treatment

3. DN led to death

End incidence of events $=$ number of endpoint incidents/ the total number of cases $\times 100 \%$.

\section{Statistical analysis}

The analyses were conducted with GraphPad Prism 5.0 and SPSS 17.0 statistical software. Data are expressed as the means \pm standard errors (SEs). For the measurement data for comparison between groups, the normality test was first performed. If the data obeyed the normal distribution, a $t$ test was used. If the data did not obey the normal distribution, the Wilcoxon rank-sum test was used. For the counting data, the $\chi^{2}$ test was used. $P<0.05$ was considered statistically significant.

\section{Results}

\section{Effects of STC-1 on renal function and morphological changes in $\mathrm{db} / \mathrm{db}$ mouse kidneys}

The $\mathrm{db} / \mathrm{db}$ mice showed increased body weights, urinary ACRs, serum creatinine levels, serum $\beta$-NAG levels, and blood glucose compared with the $\mathrm{db} / \mathrm{m}$ mice (Fig. 1a-d). In contrast, decreased urinary ACR, $\beta$-NAG levels, and blood glucose levels were observed in the $\mathrm{db} / \mathrm{db}$ mice after STC-1 treatment. Masson staining showed remarkable renal fibrosis in the kidneys of the $\mathrm{db} / \mathrm{db}$ mice, including glomerulosclerosis and interstitial fibrosis (Fig. 1g). Furthermore, TEM indicates basement membrane thickening accompanied by extensive foot process fusion (Fig. 1g). However, STC-1 treatment substantially ameliorated these changes. Quantitative analysis of the Masson-positive areas and tubular damage scores confirmed the alterations (Fig. 1f, h).

\section{STC-1 inhibits renal fibrosis in $\mathrm{db} / \mathrm{db}$ mouse kidneys}

Western blot analysis showed significantly increased FN and $\alpha$-SMA expression in the kidneys of the $\mathrm{db} / \mathrm{db}$ mice compared with the control mice (Fig. 2a-c). However, treatment with STC-1 downregulated the expression of FN and $\alpha$-SMA compared with the vehicle-treated $\mathrm{db} / \mathrm{db}$ mice. Similar results were obtained using immunohistochemical staining of FN and $\alpha$-SMA in kidney sections (Fig. 2d-f). PAS staining showed notable morphological changes in the kidneys of the db/db mice (Fig. 2d-f), including glomerular hypertrophy, increased mesangial matrix, and increased tubular epithelial disruption. However, STC-1 treatment substantially ameliorated these changes.

\section{STC-1 treatment inhibits ROS and apoptosis in $\mathrm{db} /$ db mice}

ROS generation was increased in the kidneys of the $\mathrm{db} / \mathrm{db}$ mice compared with the $\mathrm{db} / \mathrm{m}$ mice, as shown by staining with DHE (an indicator of oxidation). STC-1 treatment significantly decreased DHE staining in the $d b / d b$ mice (Fig. 3a). Compared with the control mice, the serum MDA levels were downregulated, and the serum SOD levels were upregulated. STC-1 treatment ameliorated these changes 

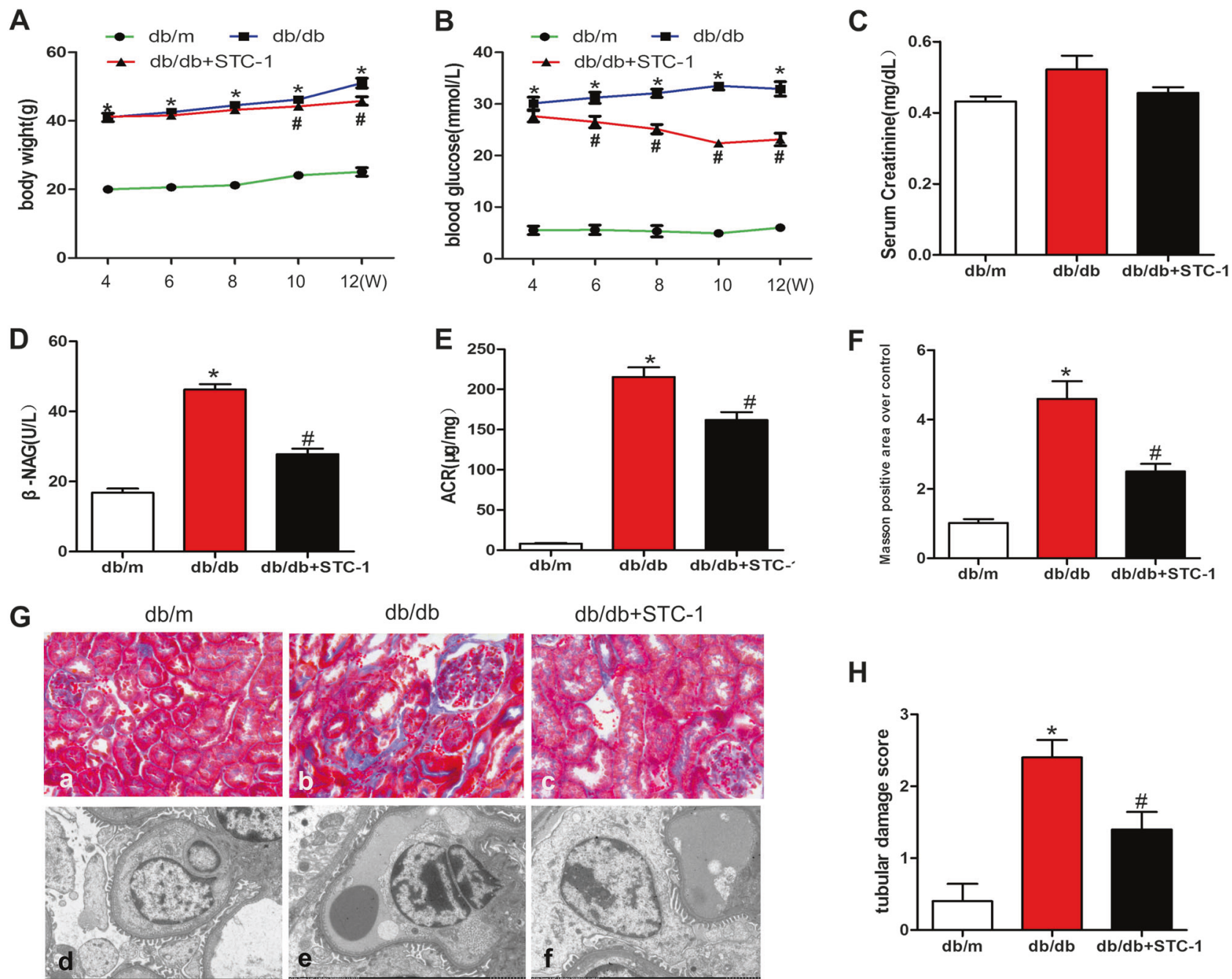

H

Fig. 1 Effects of STC-1 on renal function and morphological characteristics in $\mathrm{db} / \mathrm{db}$ mice. a Body weight changes in $\mathrm{db} / \mathrm{m}, \mathrm{db} / \mathrm{db}$, and $\mathrm{db} / \mathrm{db}$ mice that received STC-1 treatment for $4-12$ weeks. b Blood glucose concentrations in each group. c Serum creatinine levels. d Urine $\beta$-NAG levels. e Urinary ACRs. g Masson staining and EM showing notable deformations in the tubules and glomeruli of $\mathrm{db} / \mathrm{db}$ mice compared to those of $\mathrm{db} / \mathrm{m}$ mice. These changes were substantially ameliorated by STC-1 treatment. $\mathbf{f}, \mathbf{h}$ Quantitative analysis of Masson-positive staining and tubular damage in each group. Values are the mean $\pm \mathrm{SE}, * P<0.05$ vs. $\mathrm{db} / \mathrm{m} ;{ }^{\#} P<0.05$ vs. db/db mice. $n=6$
(Fig. 3c, d). Similarly, significant tubular epithelial cell apoptosis was observed via TUNEL staining in the kidneys of $\mathrm{db} / \mathrm{db}$ mice, which was attenuated by STC- 1 treatment (Fig. 3a, b). Western blot analysis showed that the ccaspase- 3 and p53 expression was significantly increased in the kidneys of the $\mathrm{db} / \mathrm{db}$ mice compared with the control mice (Fig. 3e, f). After treatment with STC-1, both ccaspase- 3 and p53 were downregulated compared with the $\mathrm{db} / \mathrm{db}$ mice.

\section{STC-1 treatment upregulates AMPK and Sirt3 and inhibits Bnip3}

Western blot analysis indicates that the p-AMPK expression was significantly reduced in the kidneys of the $\mathrm{db} / \mathrm{db}$ mice compared with the $\mathrm{db} / \mathrm{m}$ mice (Fig. 4d). Similar results were observed regarding the Sirt3 protein expression (Fig. 4a, b, $\mathrm{f}, \mathrm{h})$. In contrast, the Bnip3 protein expression was markedly increased in the kidneys of the $\mathrm{db} / \mathrm{db}$ mice (Fig. 4a, c, f, g). However, after STC-1 treatment, these changes were abrogated.

\section{STC-1 treatment attenuates high glucose-induced cell fibrosis}

Western blot analysis reveals that the $\alpha$-SMA and Fn expression was significantly upregulated in the cells treated with HG compared with the cells treated with LG (Fig. 5a). Treatment with STC-1 downregulated the expression of FN and $\alpha$-SMA. Similar results were obtained using RT-PCR 
A

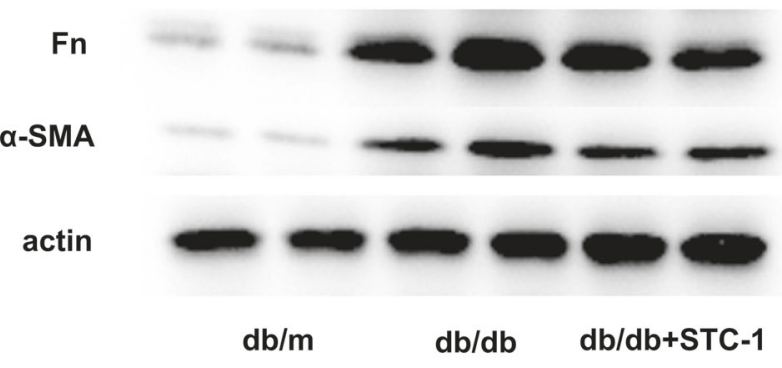

D

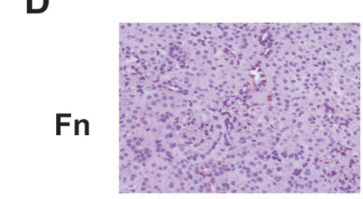

$\mathrm{db} / \mathrm{m}$

a-SMA

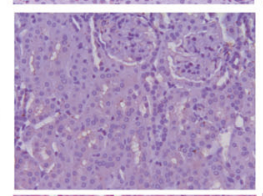

PAS

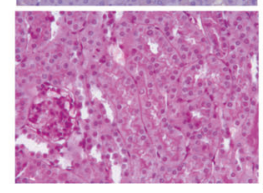

$\mathbf{d b} / \mathbf{d b}$

$\mathrm{db} / \mathrm{db}+\mathrm{STC}-1$

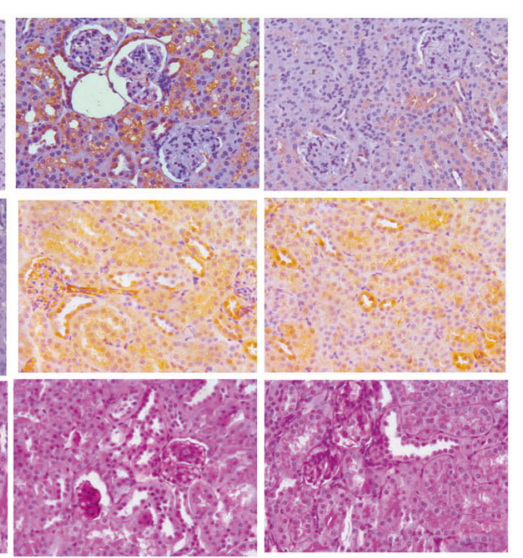

B

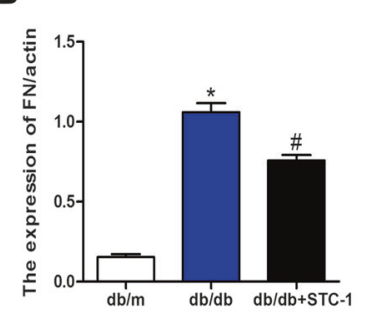

E

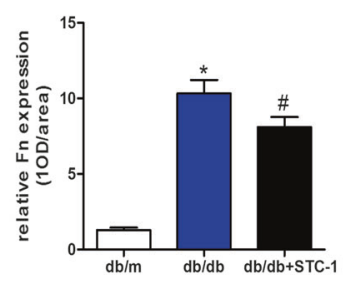

C

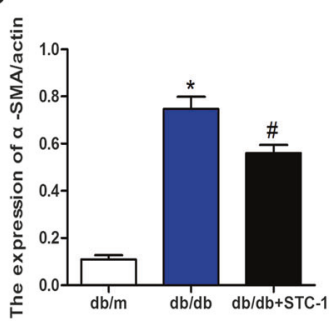

$\mathbf{F}$

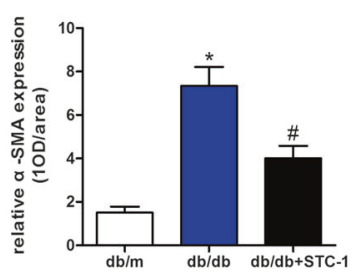

Fig. 2 STC-1 treatment inhibits renal fibrosis in $\mathrm{db} / \mathrm{db}$ mouse kidneys. Kidney samples were collected at the indicated time. a Western blot of Fn and $\alpha$-SMA. The expression of $\beta$-actin was used as a control. b Statistical analysis of the expression of FN/actin. Values are the mean $\pm \mathrm{SE}, * P<0.05$ vs. $\mathrm{db} / \mathrm{m} ;{ }^{\#} P<0.05$ vs. $\mathrm{db} / \mathrm{db}$ mice. $n=6$. c Statistical analysis of the expression of $\alpha$-SMA/actin. Values are the mean \pm SE, $* P<0.05$ vs. $\mathrm{db} / \mathrm{m} ;{ }^{\#} P<0.05$ vs. db/db mice. $n=6$. d PAS staining and immunohistochemical staining for FN and $\alpha$-SMA in kidney sections. e Relative FN expression (1OD/area). Values are the mean $\pm \mathrm{SE}, * P<0.05$ vs. $\mathrm{db} / \mathrm{m} ;{ }^{\#} P<0.05$ vs. $\mathrm{db} / \mathrm{db}$ mice. $n=6$. f Relative $\alpha$-SMA expression (1OD/area). Values are the mean $\pm \mathrm{SE}$, $* P<0.05$ vs. db/m; ${ }^{\#} P<0.05$ vs. db/db mice. $n=6$
(Figs. 5d, e). These data suggest that STC-1 inhibits HGinduced cell fibrosis.

\section{STC-1 regulates high glucose-induced p-AMPK, Sirt3, and Bnip3 expression in BUMPT cells}

Western blot analysis showed that the p-AMPK expression was significantly reduced in the HG-treated cells compared with the LG-treated cells (Fig. 6a, b). Similar results were observed regarding the Sirt3 protein or mRNA expression (Fig. 6c, d, f). In contrast, the Bnip3 protein and mRNA levels were markedly increased in the HG-treated cells (Fig. 6c, e, g). These changes were abrogated after STC-1 treatment. Similar results were seen for the in vivo experiments.

\section{STC-1 treatment inhibits ROS and apoptosis in BUMPT cells}

The MDA and SOD levels were used to evaluate the antioxidant capacity of cells. HG treatment upregulated MDA and downregulated SOD compared with the LG treatment, and STC-1 treatment ameliorated these changes, which indicates the antioxidant effect of STC-1 (Fig. 7b, c). Significant cell apoptosis was observed after DAPI staining and morphological analysis in the HG-treated cells. As shown in Fig. 7a, the affected cells displayed a typical apoptotic morphology with cell shrinkage and membrane blebbing. Moreover, staining with DAPI demonstrated chromatin condensation and nuclear fragmentation. Fortunately, the apoptosis rate was notably attenuated following STC-1 treatment (Fig. 7a). Similarly, the apoptosis detection by flow cytometry also confirmed this conclusion (Fig. 7d).

\section{Effects of AICAR, dorsomorphin, and 3-TYP on p- AMPK, Sirt3, Bnip3, p53, c-caspase3, MDA, and SOD levels in the presence of HG in BUMPT cells treated with STC-1}

To determine whether the AMPK-Sirt3 pathway modulates STC-1-induced Bnip3 expression, studies with various inhibitors or activators were performed. BUMPT cells were exposed to different concentrations of D-glucose and subjected to STC-1 treatment plus dorsomorphin (selective AMPK inhibitor), 5-aminoimidazole-4-carboxamide-1- $\beta$-D- 

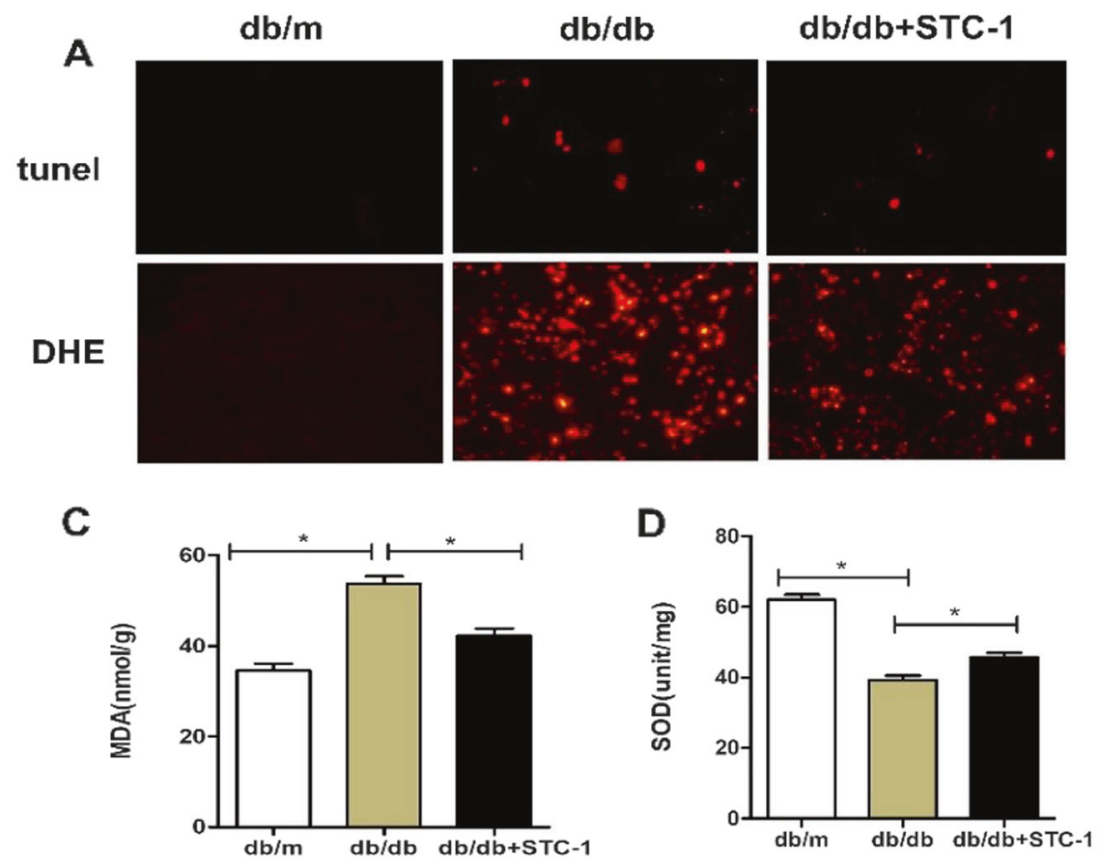

$\mathbf{E}$

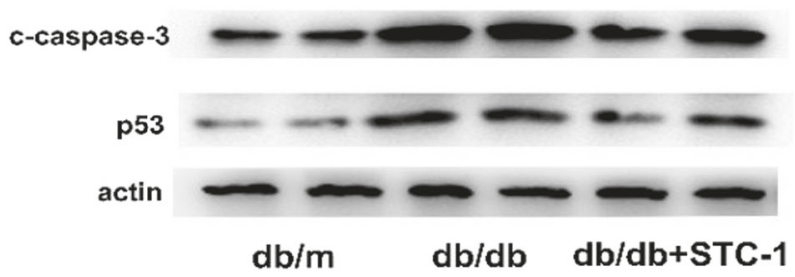

Fig. 3 STC-1 treatment inhibits ROS and apoptosis in $\mathrm{db} / \mathrm{db}$ mouse kidneys. Kidney samples were collected at the indicated time. a TUNEL and DHE staining of kidney sections $(200 \times)$. b Statistical analysis of TUNEL-positive cells. Values are the mean $\pm \mathrm{SE}, * P<$ 0.05. c Determination of MDA levels in serum by ELISA Kit. Values

ribofuranoside (AICAR; AMPK activator), or 3-TYP (selective SIRT3 inhibitor) (Fig. 8a, b, e-h). Treatment with STC-1 significantly reduced the Bnip3 expression in the BUMPT cells exposed to HG conditions, and this effect was partially blocked by dorsomorphin or 3-TYP treatment. AICAR treatment enhanced the effects of STC-1, but not in the presence of 3-TYP. Immunofluorescence showed that STC-1 treatment significantly ameliorated the decreases in the p-AMPK and Sirt3 expression elicited by HG conditions (Fig. 8a). However, these changes were nearly restored to baseline in the cells treated with dorsomorphin or 3-TYP. AICAR treatment enhanced the effects of STC-1, but not in the presence of 3-TYP. The results regarding Sirt3 contrasted with those regarding the Bnip3 expression. These findings indicate that STC-1 reduces the Bnip3 expression through the AMPK/Sirt3 pathway. These findings were also confirmed by western blot analyses (Fig. 8b). Moreover, STC-1 reduced the p53 and c-caspase 3 levels, and this
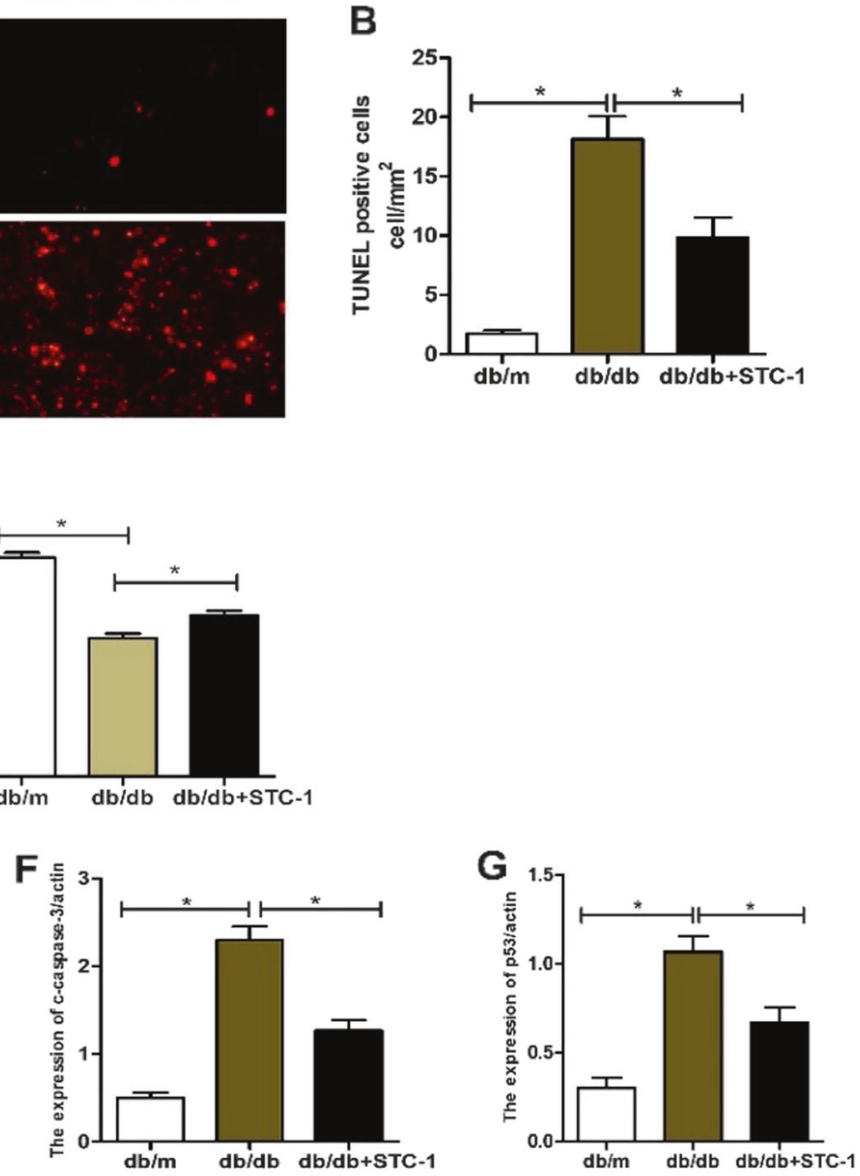

are the mean $\pm \mathrm{SE}, * P<0.05$. d Determination of SOD levels in serum by ELISA Kit. Values are the mean $\pm \mathrm{SE}, * P<0.05$. e Western blot of C-Caspase-3 and p53. The expression of $\beta$-actin was used as a control. f, $\mathbf{g}$ Statistical analysis of the expression of C-Caspase-3/actin and p53/ actin. Values are the mean $\pm \mathrm{SE}, * P<0.05$

effect was also partially blocked by dorsomorphin or 3-TYP treatment. AICAR treatment enhanced the effects of STC-1 but not in the presence of 3-TYP. Similar results were noted regarding cellular ROS injury, as determined by MDA and SOD measurements (Fig. 8c, d). Overall, these data suggest that STC-1 inhibits Bnip3 expression and ROS generation via the AMPK/Sirt3 pathway in BUMPT cells exposed to HG conditions.

\section{Follow-up completion}

As shown in Table 2, the follow-up observation time points were 12, 24, and 36 months after enrollment. After 12 months, 600 individuals remained in the cohort, and the completion ratio was $86.8 \%$. After 24 months, 512 individuals remained in the cohort, and the completion ratio was $74.1 \%$. After 36 months, 463 individuals remained in the cohort, and the completion ratio was $67.0 \%$. 

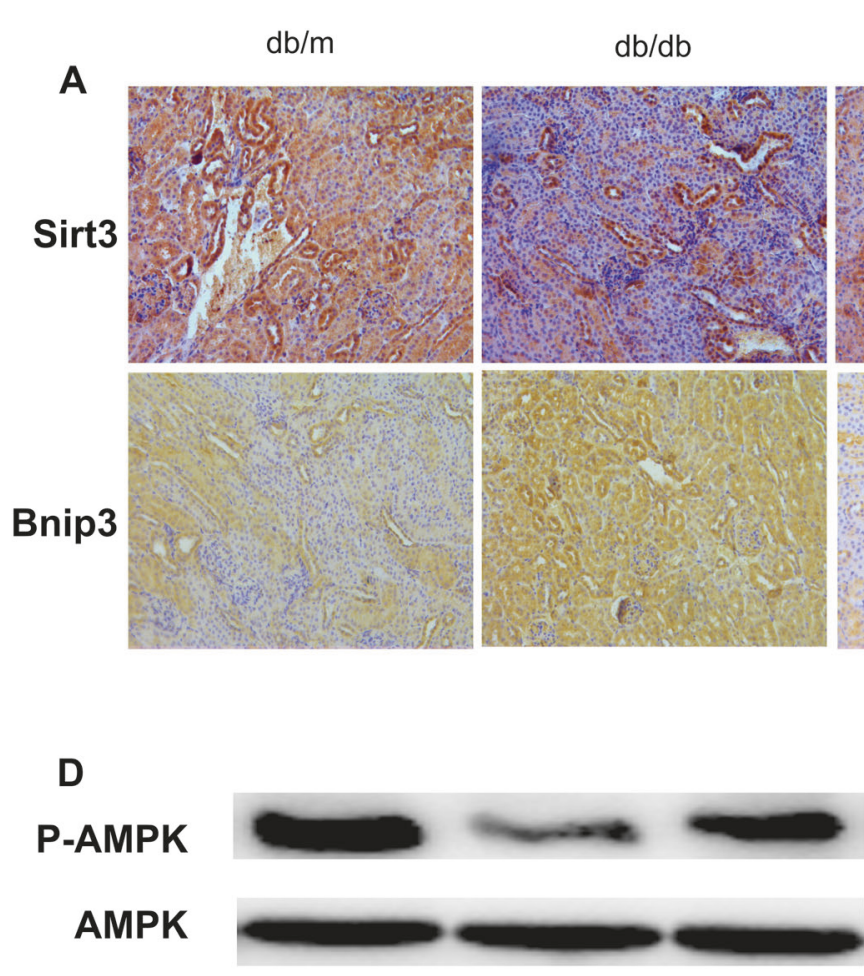

$\mathbf{F}$

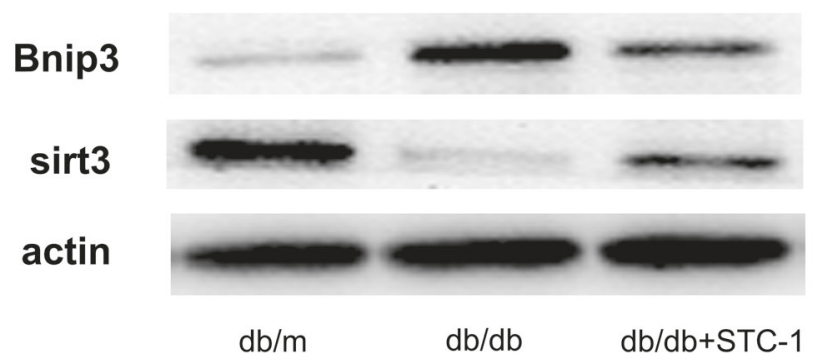

$\mathrm{db} / \mathrm{db}+\mathrm{STC}-1$

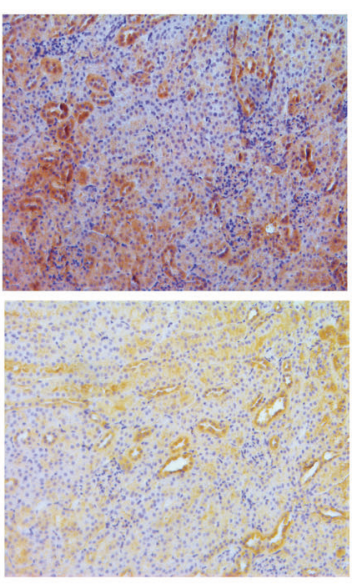

p-AMPKIAMPK

E

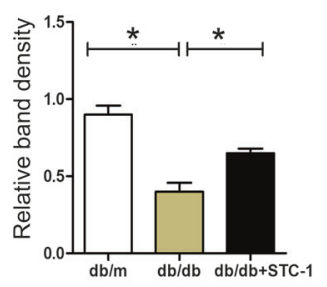

G

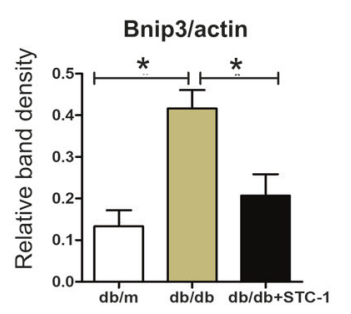

B

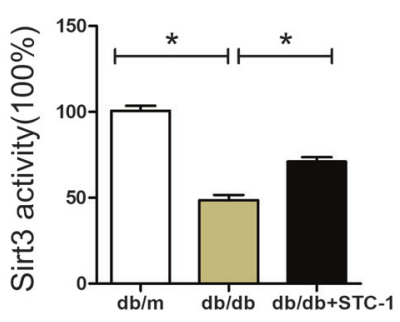

C

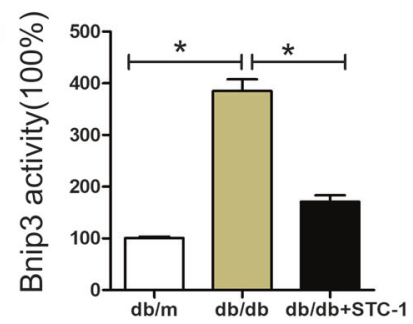

Fig. 4 STC-1 treatment upregulates AMPK and Sirt3 and inhibits Bnip3. Kidney samples were collected at the indicated time. a Immunohistochemical staining for Sirt3 and Bnip3 in kidney sections $(200 \times)$. b Relative Sirt3 expression. Values are the mean $\pm \mathrm{SE}$, ${ }^{*} P<0.05, n=6$. c Relative Bnip3 expression. Values are the mean \pm $\mathrm{SE}, * P<0.05, n=6$. d Western blot of p-AMPK. The expression of

\section{Patients with high levels of STC-1 have better prognosis}

At each time point, the number of patients who reached the endpoint event was counted, and the incidence of endpoint events was calculated. For the $<30 \mathrm{mg} / \mathrm{g}$ ACR group, if the STC-1 level was low, the incidence of endpoint events was $25.6 \%, 37.6 \%$, and $71.1 \%$ for the 12-, 24-, and 36-month time points, respectively (Table 3). In the high STC-1 level group, the incidence of endpoint events was $13.6 \%, 24.2 \%$, and $49.4 \%$ for the 12-, 24-, and 36-month time points, respectively, and there were significant differences compared with the corresponding patients in the low STC-1 group. Thus, the patients with high levels of STC-1 have a better prognosis. The same results were identified in the
AMPK was used as a control. e Statistical analysis of the expression of p-AMPK/AMPK. Values are the mean $\pm S E, * P<0.05$. f Western blot of Sirt3 and Bnip3. The expression of $\beta$-actin was used as a control. g, h Statistical analysis of the expression of Sirt3/actin and Bnip3/ actin. Values are the mean $\pm \mathrm{SE}, * P<0.05$

$30 \mathrm{mg} / \mathrm{g} \leq \mathrm{ACR}<300 \mathrm{mg} / \mathrm{g} \quad$ ACR and $\geq 300 \mathrm{mg} / \mathrm{g} \quad$ ACR groups, as shown in Tables 4 and 5.

\section{Discussion}

This study indicates that STC-1 ameliorates renal injury in $\mathrm{db} / \mathrm{db}$ mice by inhibiting ROS production and cell apoptosis mainly through the repression of Bnip3, a mitochondrial BH3-only protein that induces cell death and oxidative stress $[31,32]$. In addition, these findings suggest that STC1 inhibits Bnip3 expression via the AMPK-SIRT3 pathway. Moreover, the present study indicates that patients with high STC-1 levels have a better prognosis in each stage of DKD compared with patients with low STC-1 levels. Overall, 
A
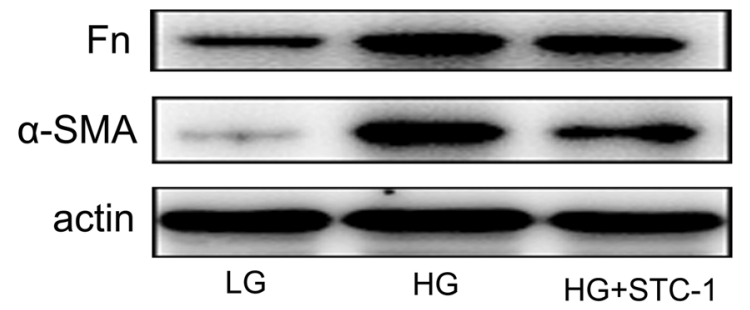

B

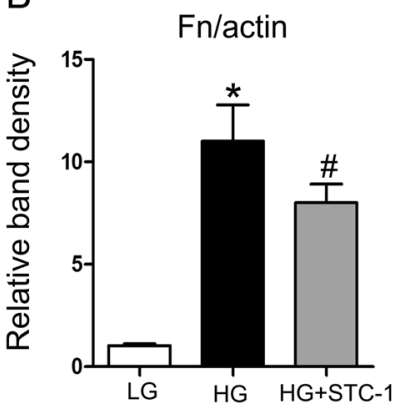

C

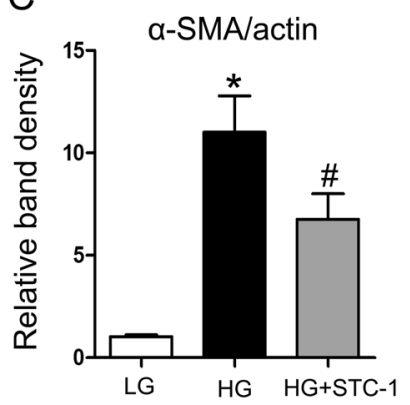

$\mathrm{D}$

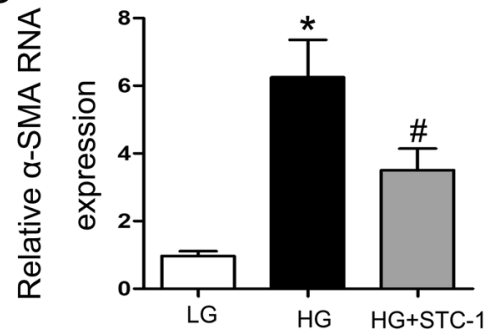

E
Fig. 5 STC-1 treatment attenuates high glucose-induced cell fibrosis. a Western blot of Fn and $\alpha$-SMA in BUMPT cells after LG treatment, HG treatment, and $\mathrm{HG}+\mathrm{STC}-1$ treatment. The expression of actin was used as a control. b, c Statistical analysis of the expression of Fn/ actin and $\alpha$-SMA/actin. Values are the mean \pm SE, $* P<0.05$, compared with LG, ${ }^{\#} P<0.05$, compared with HG. d, e RT-PCR for Fn and $\alpha$ SMA. Values are the mean $\pm \mathrm{SE}, * P<0.05$, compared with $\mathrm{LG}$, ${ }^{\#} P<0.05$, compared with $\mathrm{HG}$

\section{A \\ p-AMPK \\ AMPK}

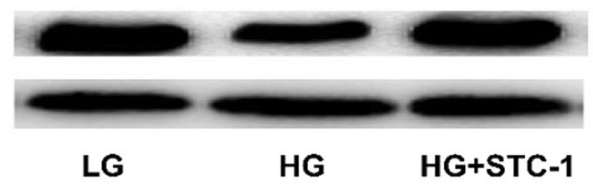

C

\section{Sirt3}

Bnip3

actin

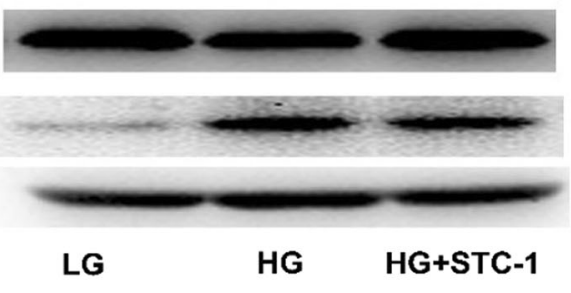

D

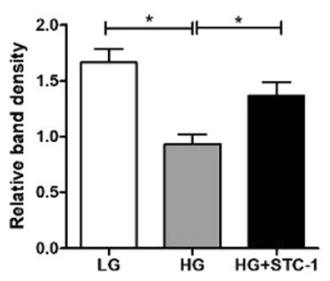

\section{E Bnip3/actin}

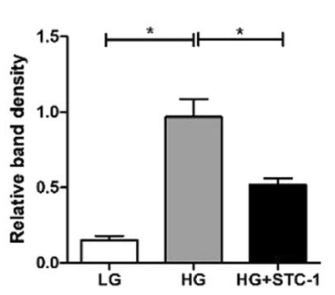

P-AMPK/AMPK

B

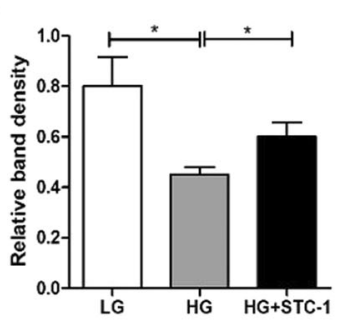

$\mathbf{F}$
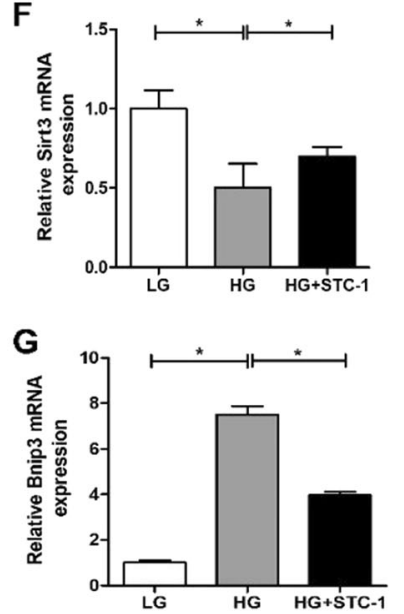

Fig. 6 STC-1 treatment upregulates AMPK and Sirt3 and inhibits Bnip3 in vitro. a Western blot of p-AMPK in BUMPT cells with different conditions (including LG treatment, HG treatment, and HG + STC-1 treatment). The expression of AMPK was used as a control. b Statistical analysis of the expression of p-AMPK/AMPK. Values are the mean $\pm \mathrm{SE}, * P<0.05$. c Western blot of Sirt3 and Bnip3 in
BUMPT cells with different conditions (including LG treatment, HG treatment, and HG + STC-1 treatment). d, e Statistical analysis of the expression of Sirt3/actin and Bnip3/actin. Values are the mean \pm SE, $* P<0.05$. f, $\mathbf{g}$ RT-PCR analysis of Sirt3 and Bnip3 in BUMPT cells with different conditions (including LG treatment, HG treatment, and $\mathrm{HG}+\mathrm{STC}-1$ treatment). Values are the mean $\pm \mathrm{SE}, * P<0.05$ 


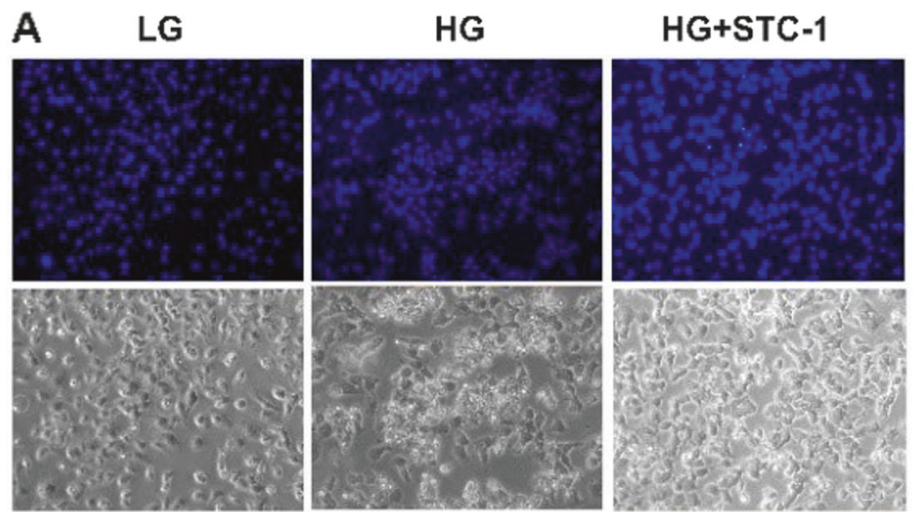

B

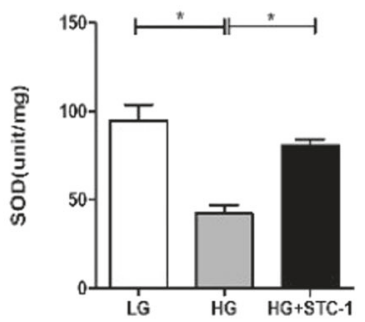

C

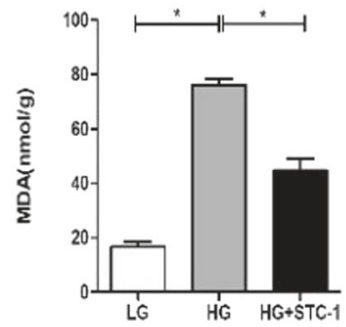

D

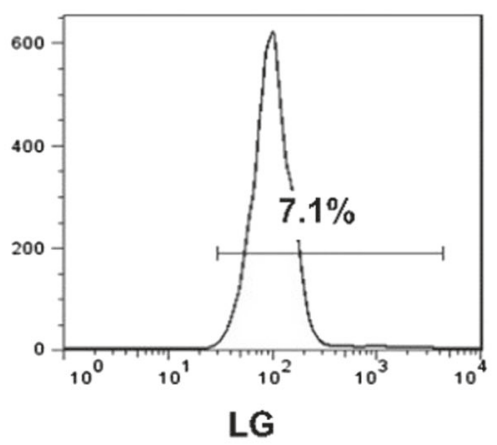

Fig. 7 STC-1 treatment inhibits ROS and apoptosis in vitro. a DAPI staining and cell morphological analysis $(200 \times)$. b Determination of MDA levels in cell lysates by ELISA Kit. Values are the mean \pm SE, $* P<0.05, n=6$. c Determination of SOD levels in cell lysates by

these results support a novel strategy for STC-1 treatment of DKD, thereby providing a new therapy option.

Intrarenal oxidative stress plays a vital role in the initiation and progression of DKD, and enhanced oxidative stress results from the overproduction of ROS. Effective agents that target the source of ROS generation hold promise to rescue kidneys from oxidative damage and prevent the subsequent progression of DKD [33, 34]. Similarly, apoptosis also contributes to DKD development, and the inhibition of apoptosis may serve as a therapeutic target in DKD [35]. Unfortunately, the mechanisms that underlie these changes have not been clearly elucidated, and no suitable drug that can reverse these changes has been identified. Bnip3 has been reported to induce apoptosis, necrosis, ROS production, or autophagy depending on the cellular context [19]. In this study, we demonstrated that Bnip3 has a role in the maintenance of ROS production and cell apoptosis and that the inhibition of Bnip3 reduces cell apoptosis and oxidative stress. Thus, therapies that suppress ROS and apoptosis may attenuate renal injury and delay the progression of DN.

In this study, the increased Bnip3 expression was related to renal oxidative damage and cell apoptosis in $\mathrm{db} / \mathrm{db}$ mice.
Another molecule that regulates Bnip3 is Sirt3, an enzyme that belongs to the family of sirtuins. Sirtuins are highly conserved NAD+-dependent deacetylases that regulate the cellular energy and lifespan in mammals through histone H3 deacetylation [36]. Upregulated Sirt3 may repress the expression of Bnip3, as well as decrease oxidative stress and cell apoptosis. A previous study has shown that Sirt3 attenuates doxorubicin-induced cardiac hypertrophy and mitochondrial dysfunction via the suppression of Bnip3 [20]. These results indicate that the overexpression of Sirt3 may ameliorate renal injury through Bnip3 inhibition, thereby reducing cell apoptosis and oxidative stress. Sirt3 is highly related to the activation of AMPK [37, 38], and AMPK phosphorylation is decreased in STZ-induced diabetic and $\mathrm{db} / \mathrm{db}$ mice, as well as in cultured mesangial cells exposed to HG [39-41]. Pan et al. demonstrated that STC-1 inhibits renal ischemia/reperfusion injury via an AMPKdependent pathway, and they also reported that AMPK regulates the STC-1-induced expression of UCP2 and sirt3, resulting in kidney protection [7]. Based on these findings, we hypothesized that STC-1 protects against renal oxidative damage and cell apoptosis by inhibiting Bnip3 expression through the AMPK/Sirt3 signaling pathway. Interestingly, 
A

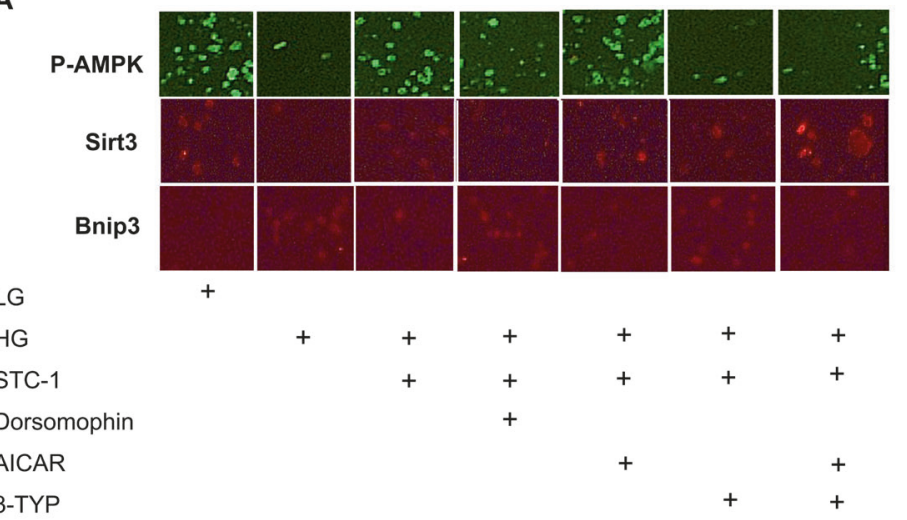

B

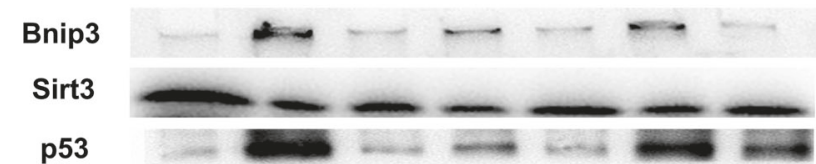

c-caspase 3

Actin

LG

HG

STC-1

Dorsomophin

AICAR

3-TYP

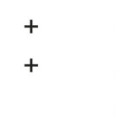

+
+
+

+
+
+

$+$

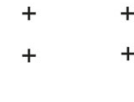

$+$

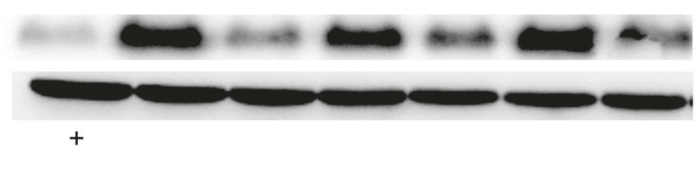

Fig. 8 Effects of AICAR, dorsomorphin, and 3-TYP on P-AMPK, Sirt3, Bnip3, p53, c-caspase3, MDA, and SOD levels under HG conditions in BUMPT cells treated with STC-1. a Cellular immunofluorescence using anti-p-AMPK, anti-Sirt3, and anti-Bnip3 antibodies. High levels of p-AMPK were observed under LG conditions and were accompanied by Sirt3 expression. HG reduced this fluorescence. This effect was reversed by STC-1, dorsomorphin, and 3-TYP treatments; however, it was increased by AICAR treatment. b Western blot showing Sirt3, Bnip3, p53, and c-caspase3 expression in BUMPT cells under HG conditions treated with or without STC-1 and

Table 2 Follow-up completion

\begin{tabular}{lll}
\hline Time & Case & Ratio (\%) \\
\hline Before follow-up & 691 & \\
Follow-up 12 months & 600 & 86.8 \\
Follow-up 24 months & 512 & 74.1 \\
Follow-up 36 months & 463 & 67.0 \\
\hline
\end{tabular}

in the present study, both the in vivo and in vitro results showed that the p-AMPK expression is significantly reduced in the kidneys of $\mathrm{db} / \mathrm{db}$ mice compared with the $\mathrm{db} /$ $\mathrm{m}$ mice. Similar results were observed regarding the Sirt3 protein expression. In contrast, Bnip3 protein expression was markedly increased in the kidneys of the $\mathrm{db} / \mathrm{db}$ mice. However, after STC-1 treatment, the levels returned to normal. We also used various inhibitors or activators in
C
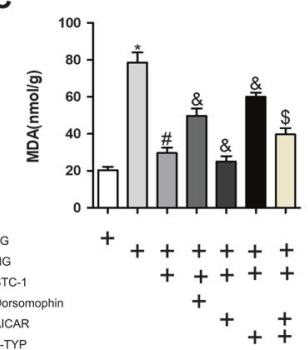

E

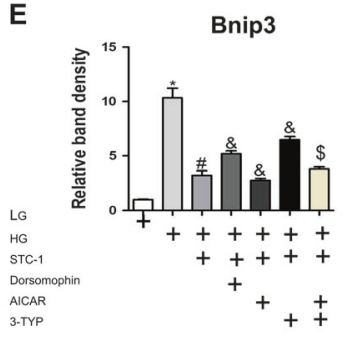

G
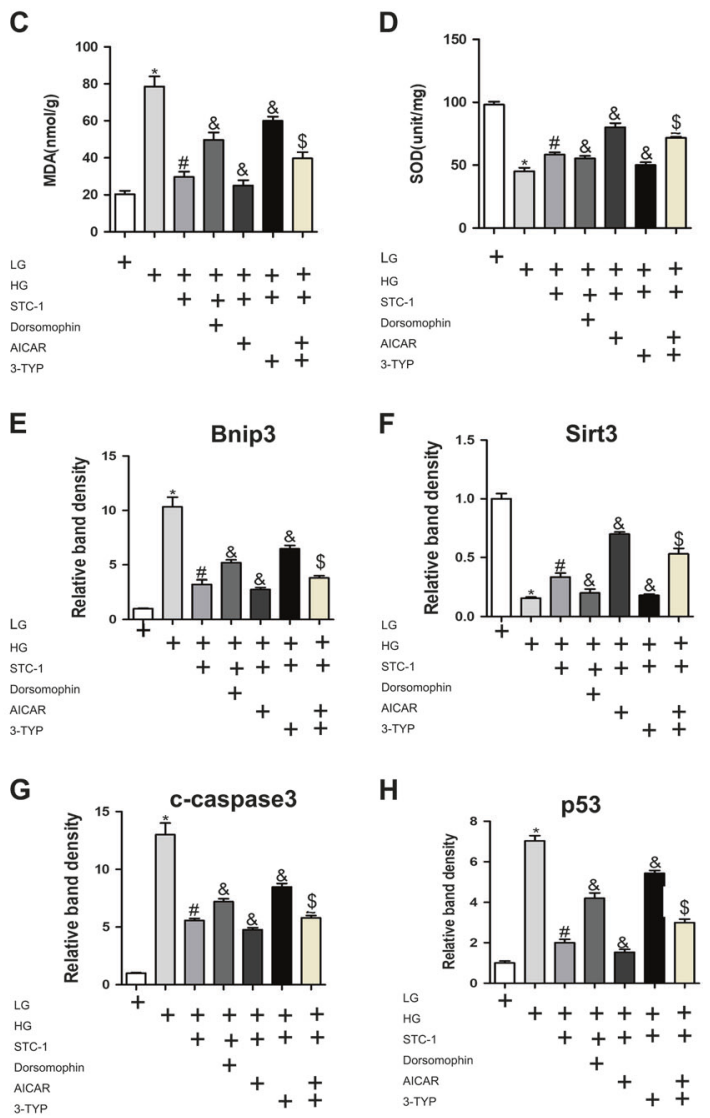

AICAR, dorsomorphin, or 3-TYP. c, d MDA and SOD levels in the medium of BUMPT cells. Values are the mean $\pm \mathrm{SE}(n=4), * P<0.01$ vs. LG; ${ }^{\#} P<0.01$ vs. $\mathrm{HG} ;{ }^{\&} P<0.01$ vs. $\mathrm{HG}+\mathrm{STC}-1 .{ }^{\$} P<0.01$ vs. HG + STC-1 + 3-TYP. e-h Statistical analysis of the expression of Bnip3, Sirt3, c-caspase3, and p53 in BUMPT cells exposed to HG conditions with or without treatment with various reagents. Quantitative analyses of western blotting results, and data were normalized to $\beta$-actin. Data are presented as the mean $\pm \mathrm{SE}(n=4)$. ${ }^{*} P<0.01$ vs. LG; ${ }^{\#} P<0.01$ vs. HG; ${ }^{\&} P<0.01$ vs. $\mathrm{HG}+\mathrm{STC}-1 ;{ }^{\$} P<0.01$ vs. $\mathrm{HG}+\mathrm{STC}-1+3-\mathrm{TYP}$

BUMPT cells exposed to different concentrations of Dglucose, as well as treated with STC-1 plus dorsomorphin (selective AMPK inhibitor), 5-aminoimidazole-4-carboxamide-1- $\beta$-D-ribofuranoside (AICAR; AMPK activator), or 3-TYP (selective SIRT3 inhibitor). Treatment with STC-1 significantly reduced the Bnip3 expression in BUMPT cells exposed to $\mathrm{HG}$ conditions, and this effect was partially blocked by the dorsomorphin and 3-TYP treatments. AICAR treatment enhanced the effects of STC-1 but not in the presence of 3-TYP. These findings suggest that STC-1 reduces the Bnip3 expression through the AMPK/Sirt3 pathway.

To investigate the relationship between the STC-1 level and the prognosis of DKD patients, we conducted a prospective cohort study that consisted of 691 patients diagnosed with DKD. The prognosis between a low STC-1 level and high STC-1 level in each group had significant 
Table 3 Incidence of endpoint events in $\mathrm{ACR}<30 \mathrm{mg} / \mathrm{g}$ group

\begin{tabular}{llllll}
\hline ACR $<30(\mathrm{mg} / \mathrm{g})$ & STC-1 levels & Time & Case & Case-positive & Ratio (\%) \\
\hline \multirow{2}{*}{ Low } & Before follow-up & 99 & & \\
& & Follow-up 12 months & 90 & 23 & 25.6 \\
& Follow-up 24 months & 85 & 32 & 37.6 \\
& Follow-up 36 months & 76 & 54 & 71.1 \\
& High & Before follow-up & 117 & & $13.6^{*}$ \\
& Follow-up 12 months & 110 & 15 & $24.2^{\#}$ \\
& Follow-up 24 months & 99 & 24 & $49.4^{\&}$ \\
\hline
\end{tabular}

${ }^{*} P<0.05$, compared with low STC-1 group when follow-up was 12 months; ${ }^{\#} P<0.05$, compared with low STC-1 group when follow-up was 24 months; ${ }^{\&} P<0.05$, compared with low STC-1 group when follow-up was 36 months

\begin{tabular}{llllll}
\hline $30<\mathrm{ACR}<300(\mathrm{mg} / \mathrm{g})$ & STC-1 levels & Time & Case & Case-positive & Ratio (\%) \\
\hline \multirow{2}{*}{ Low } & Before follow-up & 112 & & \\
& & Follow-up 12 months & 101 & 42 & 41.6 \\
& Follow-up 24 months & 92 & 61 & 66.3 \\
& High & Follow-up 36 months & 78 & 70 & 89.7 \\
& Before follow-up & 91 & & $36.5^{*}$ \\
& Follow-up 12 months & 85 & 31 & $52.5^{*} \#$ \\
& Follow-up 24 months & 80 & 42 & $68.9 * \&$
\end{tabular}

${ }^{*} P<0.05$, compared with low STC-1 group when follow-up was 12 months; ${ }^{*} P<0.05$, compared with low STC-1 group when follow-up was 24 months; ${ }^{\&} P<0.05$, compared with low STC-1 group when follow-up was 36 months

Table 4 Incidence of endpoint events in $30 \mathrm{mg} / \mathrm{g} \leq \mathrm{ACR}<300$ $\mathrm{mg} / \mathrm{g}$ group

Table 5 Incidence of endpoint events in ACR $\geq 30 \mathrm{mg} / \mathrm{g}$ group

\begin{tabular}{llllll}
\hline AC $\geq 300(\mathrm{mg} / \mathrm{g})$ & STC-1 levels & Time & Case & Case-positive & Ratio (\%) \\
\hline \multirow{2}{*}{ Low } & Before follow-up & 140 & & \\
& & Follow-up 12 months & 114 & 63 & 55.3 \\
& Follow-up 24 months & 79 & 54 & 68.4 \\
& Follow-up 36 months & 78 & 63 & 80.1 \\
& High & Before follow-up & 132 & & \\
& & Follow-up 12 months & 100 & 49 & $49 *$ \\
& Follow-up 24 months & 77 & 35 & $45.5 * \#$ \\
& Follow-up 36 months & 73 & 43 & $60 * \&$ \\
\hline
\end{tabular}

${ }^{*} P<0.05$, compared with low STC-1 group when follow-up was 12 months; ${ }^{*} P<0.05$, compared with low STC-1 group when follow-up was 24 months; ${ }^{\&} P<0.05$, compared with low STC-1 group when follow-up was 36 months differences. These results suggest that DKD patients with high levels of STC-1 have a better prognosis, which indicates that the serum STC-1 is independently associated with DKD progression in type 2 diabetic patients. Thus, STC-1 may be a novel factor that plays a role in the pathophysiology of DKD. Unfortunately, the present findings do not indicate a biological link between higher serum STC-1 levels and DKD progression in the clinic.
In summary, the present study indicates that STC-1 ameliorates oxidative stress and cell apoptosis in the kidneys of the $\mathrm{db} / \mathrm{db}$ mice and HG-treated BUMPT cells by inhibiting Bnip3 expression, which is mediated by activating the AMPK/Sirt3 pathway. The clinical data show that serum STC-1 is independently associated with DKD progression in patients with type 2 diabetes and that patients with high levels of STC-1 have a better prognosis. Thus, 
synthetic STC-1 is a potential drug candidate for the treatment of DKD patients. However, other studies have shown that STC-1 may serve as a damaging factor. For example, increased STC-1 expression has been shown to be closely associated with several cancers and poor prognostic outcomes [7]. Therefore, additional randomized doubleblinded human trials must be conducted to determine whether STC-1 is a safe and useful option for DKD treatment.

Acknowledgements This study was funded by research grants (81870500, 81770714) from the National Natural Science Foundation of China. It was also supported by the research grants (2017JJ2002) from the Natural Science Foundation of Hunan Province, a research grant from Health and Family Planning Commission of Hunan Province (20180922), and a research grant from the Changde Municipal Science and Technology Bureau (2016KZ34).

\section{Compliance with ethical standards}

Conflict of interest The authors declare that they have no conflict of interest.

Publisher's note: Springer Nature remains neutral with regard to jurisdictional claims in published maps and institutional affiliations.

\section{References}

1. Lin YC, Chang YH, Yang SY, Wu KD, Chu TS. Update of pathophysiology and management of diabetic kidney disease. J Formos Med Assoc. 2018;117:662-75.

2. Liang S, Cai GY, Chen XM. Clinical and pathological factors associated with progression of diabetic nephropathy. Nephrol (Carlton). 2017;22(Suppl 4):14-19.

3. Yuan S, Liu X, Zhu X, Qu Z, Gong Z, Li J, et al. The role of TLR4 on PGC-1alpha-mediated oxidative stress in tubular cell in diabetic kidney disease. Oxid Med Cell Longev. 2018;2018:6296802.

4. Xiao L, Xu X, Zhang F, Wang M, Xu Y, Tang D, et al. The mitochondria-targeted antioxidant MitoQ ameliorated tubular injury mediated by mitophagy in diabetic kidney disease via Nrf2/ PINK1. Redox Biol. 2017;11:297-311.

5. Yoshiko Y, Aubin JE, Maeda N. Stanniocalcin 1 (STC1) protein and mRNA are developmentally regulated during embryonic mouse osteogenesis: the potential of stc1 as an autocrine/paracrine factor for osteoblast development and bone formation. J Histochem Cytochem. 2002;50:483-92.

6. Sheikh-Hamad D. Mammalian stanniocalcin-1 activates mitochondrial antioxidant pathways: new paradigms for regulation of macrophages and endothelium. Am J Physiol Ren Physiol. 2010;298:F248-254.

7. Su J1, Guo B, Zhang T, Wang K, Li X, Liang G. Stanniocalcin-1, a new biomarker of glioma progression, is associated with prognosis of patients. Tumour Biol. 2015;36:6333-9.

8. Liu D, Shang H, Liu Y. Stanniocalcin-1 protects a mouse model from renal ischemia-reperfusion injury by affecting ROSmediated multiple signaling pathways. Int $\mathrm{J}$ Mol Sci. 2016;17:1-19

9. Pan JS, Huang L, Belousova T, Lu L, Yang Y, Reddel R, et al. Stanniocalcin-1 inhibits renal ischemia/reperfusion injury via an
AMP-activated protein kinase-dependent pathway. J Am Soc Nephrol. 2015;26:364-78.

10. Bause AS, Haigis MC. SIRT3 regulation of mitochondrial oxidative stress. Exp Gerontol. 2013;48:634-9.

11. Koentges C, Bode C, Bugger H. SIRT3 in cardiac physiology and disease. Front Cardiovasc Med. 2016;3:38.

12. Yuan C, Pu L, He Z, Wang J. BNIP3/Bcl-2-mediated apoptosis induced by cyclic tensile stretch in human cartilage endplatederived stem cells. Exp Ther Med. 2018;15:235-41.

13. Dhingra R, Margulets V, Chowdhury SR, Thliveris J, Jassal D, Fernyhough $\mathrm{P}$, et al. Bnip3 mediates doxorubicin-induced cardiac myocyte necrosis and mortality through changes in mitochondrial signaling. Proc Natl Acad Sci USA. 2014;111:E5537-5544.

14. Ghavami S, Eshraghi M, Kadkhoda K, Mutawe MM, Maddika S, Bay GH, et al. Role of BNIP3 in TNF-induced cell death-TNF upregulates BNIP3 expression. Biochim Biophys Acta. 2009; 1793:546-60.

15. Regula KM, Ens K, Kirshenbaum LA. Inducible expression of BNIP3 provokes mitochondrial defects and hypoxia-mediated cell death of ventricular myocytes. Circ Res. 2002;91:226-31.

16. Wang EY, Gang H, Aviv Y, Dhingra R, Margulets V, Kirshenbaum LA. p53 mediates autophagy and cell death by a mechanism contingent on Bnip3. Hypertension. 2013;62:70-77.

17. Diwan A, Krenz M, Syed FM, Wansapura J, Ren X, Koesters AG, et al. Inhibition of ischemic cardiomyocyte apoptosis through targeted ablation of Bnip3 restrains postinfarction remodeling in mice. J Clin Invest. 2007;117:2825-2833.

18. Li Y, Zhang X, Yang J, Zhang Y, Zhu D, Zhang L, et al. Methylation of BNIP3 in pancreatic cancer inhibits the induction of mitochondrial-mediated tumor cell apoptosis. Oncotarget. 2017;8:63208-22.

19. Moriyama M, Moriyama H, Uda J, Kubo H, Nakajima Y, Goto A, et al. BNIP3 upregulation via stimulation of ERK and JNK activity is required for the protection of keratinocytes from UVBinduced apoptosis. Cell Death Dis. 2017;8:e2576.

20. Du Q, Zhu B, Zhai Q, Yu B. Sirt3 attenuates doxorubicin-induced cardiac hypertrophy and mitochondrial dysfunction via suppression of Bnip3. Am J Transl Res. 2017;9:3360-73.

21. He L, Peng X, Zhu J, Chen X, Liu H, Tang C, et al. Mangiferin attenuate sepsis-induced acute kidney injury via antioxidant and anti-inflammatory effects. Am J Nephrol. 2014;40:441-50.

22. Mohammadipoor A, Lee RH, Prockop DJ, Bartosh TJ. Stanniocalcin-1 attenuates ischemic cardiac injury and response of differentiating monocytes/macrophages to inflammatory stimuli. Transl Res. 2016;177:127-42.

23. Livingston MJ, Ding HF, Huang S, Hill JA, Yin XM, Dong Z. Persistent activation of autophagy in kidney tubular cells promotes renal interstitial fibrosis during unilateral ureteral obstruction. Autophagy. 2016;12:976-98.

24. Yu PB, Hong CC, Sachidanandan C, Babitt JL, Deng DY, Hoyng $\mathrm{SA}$, et al. Dorsomorphin inhibits BMP signals required for embryogenesis and iron metabolism. Nat Chem Biol. 2008;4:33-41.

25. Andersen C, Kotowska D, Tortzen CG, Kristiansen K, Nielsen J, Petersen RK. 2-(2-Bromophenyl)-formononetin and 2-heptylformononetin are PPARgamma partial agonists and reduce lipid accumulation in 3T3-L1 adipocytes. Bioorg Med Chem. 2014;22:6105-11.

26. Zeng Z, Yang Y, Dai X, Xu S, Li T, Zhang Q, et al. Polydatin ameliorates injury to the small intestine induced by hemorrhagic shock via SIRT3 activation-mediated mitochondrial protection. Expert Opin Ther Targets. 2016;20:645-52.

27. Pi H, Xu S, Reiter RJ, Guo P, Zhang L, Li Y, et al. SIRT3-SOD2mROS-dependent autophagy in cadmium-induced hepatotoxicity and salvage by melatonin. Autophagy. 2015;11:1037-51. 
28. Yang S, Zhao L, Han Y, Liu Y, Chen C, Zhan M, et al. Probucol ameliorates renal injury in diabetic nephropathy by inhibiting the expression of the redox enzyme p66Shc. Redox Biol. 2017;13:482-97.

29. Peng X, Yang T, Liu G, Liu H, Peng Y, He L. Piperine ameliorated lupus nephritis by targeting AMPK-mediated activation of NLRP3 inflammasome. Int Immunopharmacol. 2018;65:448-57.

30. Liu JJ, Pek SLT, Ang K, Tavintharan S, Lim SC. Plasma leucinerich alpha-2-glycoprotein 1 predicts rapid eGFR decline and albuminuria progression in type 2 diabetes mellitus. J Clin Endocrinol Metab. 2017;102:3683-91.

31. Zhang T, Xue L, Li L, Tang C, Wan Z, Wang R, et al. BNIP3 protein suppresses PINK1 kinase proteolytic cleavage to promote mitophagy. J Biol Chem. 2016;291:21616-29.

32. Liu KE, Frazier WA. Phosphorylation of the BNIP3 C-terminus inhibits mitochondrial damage and cell death without blocking autophagy. PLoS One. 2015;10:e129667.

33. Jha JC, Banal C, Chow BS, Cooper ME, Jandeleit-Dahm K. Diabetes and kidney disease: role of oxidative stress. Antioxid Redox Signal. 2016;25:657-84.

34. Liu YN, Zhou J, Li T, Wu J, Xie SH, Liu HF, et al. Sulodexide protects renal tubular epithelial cells from oxidative stressinduced injury via upregulating klotho expression at an early stage of diabetic kidney disease. J Diabetes Res. 2017;2017:4989847.

35. Zou HH, Yang PP, Huang TL, Zheng XX, Xu GS. PLK2 plays an essential role in high D-glucose-induced apoptosis, ROS generation and inflammation in podocytes. Sci Rep. 2017;7:4261.

36. Zhang T, Kraus WL. SIRT1-dependent regulation of chromatin and transcription: linking $\mathrm{NAD}(+)$ metabolism and signaling to the control of cellular functions. Biochim Biophys Acta. 2010;1804:1666-75.

37. Huh JE, Shin JH, Jang ES, Park SJ, Park DR, Ko R1, et al. Sirtuin 3 (SIRT3) maintains bone homeostasis by regulating AMPKPGC-1beta axis in mice. Sci Rep. 2016;6:22511.

38. Fu J, Jin J, Cichewicz RH, Hageman SA, Ellis TK, Xiang L, et al. trans-(-)-epsilon-Viniferin increases mitochondrial sirtuin 3 (SIRT3), activates AMP-activated protein kinase (AMPK), and protects cells in models of Huntington disease. J Biol Chem. 2012;287:24460-72.

39. Guo Y, Yu W, Sun D, Wang J, Li C, Zhang R, et al. A novel protective mechanism for mitochondrial aldehyde dehydrogenase (ALDH2) in type i diabetes-induced cardiac dysfunction: role of AMPK-regulated autophagy. Biochim Biophys Acta. 2015;1852:319-31.

40. Guo YN, Wang JC, Cai GY, Hu X, Cui SY, Lv Y, et al. AMPKmediated downregulation of connexin 43 and premature senescence of mesangial cells under high-glucose conditions. Exp Gerontol. 2014;51:71-81.

41. Hong YA, Lim JH, Kim MY, Kim TW, Kim Y, Yang KS, et al. Fenofibrate improves renal lipotoxicity through activation of AMPK-PGC-1alpha in db/db mice. PLoS One. 2014;9: e96147. 